\title{
Genotypic and Serotypic confirmations of Bacterial community to Kotumsar cave for occupational safety of cave workers and visitors from pathogenic threats
}

\begin{abstract}
:
Background: Bacterial communities exist everywhere in the universe so in the caves. Kotumsar cave is the one of the renowned tourist place in India therefore high risk of anthropogenic pressure in this ecological niche. Objective: In the present study some pathogenic and nonpathogenic soil bacterial communities earlier isolated and characterized from different microhabitats of Kotumsar cave have been further confirmed by using serological and molecular technique i.e. applying Amplified Ribosomal DNA Restriction Analysis (ARDRA). Methods: Identified bacterial cultures were re-cultured for 16SrDNA analysis. Cluster analysis was used to analyze the restriction pattern generated by the enzyme by the using NTSYS-pc and UPGMA software and as well serotyping is done by Prescott's method. Result: Total twelve bacteria were selected for serological and molecular characterization. There were two most suitable enzymes $E c o R I$ and Alul which shows typical molecular characteristics between the similar genuses. Conclusion: This piece of work confirms some pathogenic bacteria in Kotumsar cave's habitat. Though they are not highly pathogenic but repeated visiting in this cave environment may cause infectivity accordingly proper safety precaution must followed after visiting the cave.
\end{abstract}

Key Words: Cave, Bacterial isolates, $16 \mathrm{~S}$ rDNA, ARDRA, Restriction enzyme, Serotyping .

\section{Introduction}

Subterranean biospheres are usually characterized by high humidity, complete darkness and low energy inputs. Kotumsar Cave is one of the most biologically explored limestone caves of India, time to time several troglobiotic as well as troglophilic species have been identified from this cave. It is situated in the Kanger Valley National Park $\left(18^{\circ} 52^{\prime} 09^{\prime \prime} \mathrm{N}\right.$; $\left.81^{\circ} 56^{\prime} 05^{\prime \prime} \mathrm{E}\right)$, which became a major eco-tourist spot of Chhattisgarh, after it has been developed as a new state of India. The main entrance of this cave is formed by a vertical fissure in the wall of a hill which further leads inwards via a narrow, twisted tubular path, measuring about $15 \mathrm{~m}$ in length. The cave is honeycombed in its structure, consisting of several irregular chambers. The main tunnel of the cave is nearly $500 \mathrm{~m}$ long and has several lateral and downward passages. The roofs and walls of the different chambers are lined with colorful dripstone formations resulting from the precipitation of calcite-dissolved carbonate lime. The chambers of the cave are floored with either rocks or pebbles of various dimensions or by surface-derived soil/clay deposits. The cave is subject to frequent flooding during the monsoon season which generally begins in the middle of
June and continues till the mid of October. The air and water temperature of the cave remain relatively stable at an annual average of $28.25 \pm 1.23$ and $26.33 \pm 0.96^{\circ} \mathrm{C}$ respectively (range $=25 \cdot 0-32 \cdot 7^{\circ} \mathrm{C}$ for air; $22 \cdot 9-29 \cdot 3^{\circ} \mathrm{C}$ for water).

Earlier we succeeded to isolate total one forty six bacterial strain from the various microhabitat soil sediments of Kotumsar cave. The development and use of modern technologies like PCR amplification for fast examination and screening of activities as well as determining the composition of microbial community [10]. ARDRA, as a simple method based on restriction endonuclease digestion of the amplification fragment of bacterial 16S rDNA, gives only a little information about the type of microorganism present in the sample, but allows to search for changes in the community over time, or even to compare those changes to current environmental condition [4]. In the present study an attempt has been taken rectify the same strain by employing an Amplified Ribosomal DNA Restriction Analysis (ARDRA) technique, the characterization based on molecular analysis technique and also serological confirmation. 


\section{Methods}

Earlier identified bacterial species were re-cultured on nutrient agar medium at $28 \pm 1^{\circ} \mathrm{C}$ and maintained by sub-culturing every fortnightly at $4 \mathrm{oC}$ for employing molecular characterization.

\section{Serotyping of bacteria}

Serology is a method to investigate the molecular architecture of the bacterial cell, depend on the ability of the chemical constituents of the same to behave as antigens, i.e. to elicit the production of antibodies in vertebrate animals. Live antigen was used to raise antiserum against Micrococcus luteus3. The cell concentration was $7^{\prime} 10^{10} \mathrm{CFU} / \mathrm{ml}$. Rabbit was immunized as per the Prescott, 1981 [7]. For agglutination 100ml bacterial suspension and $100 \mathrm{ml}$ of polyclonal antibody were placed on slide. Preimmunized serum and saline were used as a control.

\section{Amplified Ribosomal DNA Restriction Analysis (ARDRA)}

For ARDRA, the genomic DNA was extracted by the following method of Sambrook, et. Al [12]. Oligonucleotide primer was derived from conserved region present at the edges of the $16 \mathrm{~S}$ rDNA. The sequences of primers were 16SrDNA F- 5' AGAGTTTGATCCTGGCTCAG-3' and 16SrDNA R-5'AGGAGGTGATCCAGCCGCA-3' [14]. The template DNA ( $1 \mu \mathrm{l}$, around $10 \mathrm{ng}$ DNA) was added to $49 \mu$ l aliquots of PCR mixture containing $5 \mu$ of $10 X$ PCR buffer supplied with enzyme (Taq polymerase), $2.5 \mu \mathrm{l}$ each forward and reverse primer from a 10 pico mol stock (i.e. 12.5 pico mol of each primer), $2.5 \mu \mathrm{l}$ of $10 \mathrm{mM}$ (each) deoxynucleoside triphosphates, and $0.5 \mu \mathrm{l}$ of DNA polymerase ( 3 unit/ml) final volume was made to $50 \mu$ by adding sterile glass distilled water. After initial denaturation at $74^{\circ} \mathrm{C}$ for 3 $\mathrm{min}$, the reaction mixture was run through 35 cycles of denaturation at $94^{\circ} \mathrm{C}$ for $1 \mathrm{~min}$, annealing at $51^{\circ} \mathrm{C}$ for $1 \mathrm{~min}$, and finally a 10 min extension period at $72^{\circ} \mathrm{C}$ was carried out. The $16 \mathrm{~S}$ rRNA type was determined by digestion of the amplicon with EcoRI (G/ AATTC) and Alul (AG/CT) enzymes (Banglore Genei, India) and analyzed by electrophoresis on $1.5 \%$ agarose gel with staining by ethidium bromide $(0.5 \mathrm{mg} / \mathrm{ml})$. ARDRA bands were scored as either present (1) or absent (0). All binary data were entered and genetic distances were calculated through Numerical Taxonomy and Multivariate Analysis System (NTSYS-pc), version 2.02 and calculating Euclidean distance and then assembling a dendogram using "Unweighted Paired Group Method using Arithmetic average criterion" (UPGMA).

\section{Results}

The immune sera from rabbit when subjected to direct agglutination with the Kotumsar's bacterial isolates were non-specific, as shown in table 1 , when serum reacted with its homologous bacterial isolate by agglutination. All fourteen bacterial isolates were tested by agglutination and double diffusion, using a soluble, particulate and sonicated antigen. Further, only nine Micrococcus sps. exhibited agglutination with antisera of Micrococcus luteus3, but rest of the isolates did not show agglutination. However, sonicated antigen and soluble antigen of bacteria did not show any precipitation through double diffusion. Two isolates of Stomatococcus mucilaginosus KCB26 \& KCB14, Micrococcus luteus $4 \mathrm{KCB} 11$ and Deinococcus radiodurans KCB 21 from entrance zone whereas Micrococcus luteus $3 \mathrm{KCB} 38$ from twilight zone, Deinococcus radiodurans KCB50 from transient zone likewise Micrococcus agilis KCB125 from foval soil, Deinococcus radiodurans KCB 93 from guano mixed soil and also Micrococcus agilis KCB140 from red laterite soil of deep zone exhibited agglutination with antisera. Therefore, serological relationship exists between these organisms. Rest of the three bacterial isolates Staphylococcus simulans KCB129, Staphylococcus varians KCB134 from deep zone (foval soil), and Streptococcus grp Q1 KCB109 from deep zone (guano mix soil) did not show agglutination reaction.

Table 1: Serotyping of bacteria from soil of Kotumsar cave by agglutination reaction

\begin{tabular}{|c|c|c|c|c|}
\hline $\begin{array}{l}\text { S. } \\
\text { N. }\end{array}$ & Zone & $\begin{array}{l}\text { Culture } \\
\text { code }\end{array}$ & $\begin{array}{l}\text { Aggluti- } \\
\text { nation } \\
\text { reaction }\end{array}$ & Name of the bacteria \\
\hline \multirow{4}{*}{1.} & \multirow{4}{*}{$\begin{array}{l}\text { Entrance } \\
\text { zone }\end{array}$} & кСВ26 & + & $\begin{array}{l}\text { Stomatococcus } \\
\text { mucilaginosus }\end{array}$ \\
\hline & & КСВ21 & + & $\begin{array}{l}\text { Deinococcus } \\
\text { radiodurans }\end{array}$ \\
\hline & & KCB14 & + & $\begin{array}{l}\text { Stomatococcus } \\
\text { mucilaginosus }\end{array}$ \\
\hline & & ксв11 & +++ & Micrococcus luteus4 \\
\hline 2. & $\begin{array}{l}\text { Twilight } \\
\text { zone }\end{array}$ & кСв38 & ++++ & Micrococcus luteus 3 \\
\hline 3. & $\begin{array}{l}\text { Transient } \\
\text { zone }\end{array}$ & KCB50 & + & Deinococcus radidurans \\
\hline \multirow{3}{*}{4.} & \multirow{3}{*}{$\begin{array}{l}\text { Deep zone } \\
\text { (foval soil) }\end{array}$} & КСВ134 & - & Staphylococcus varians \\
\hline & & КСB125 & + & Micrococcus agilis \\
\hline & & КСВ129 & - & $\begin{array}{l}\text { Staphylococcus } \\
\text { simulans }\end{array}$ \\
\hline 5. & $\begin{array}{l}\text { Deep zone } \\
\text { (guano mix } \\
\text { soil) }\end{array}$ & кСВ109 & - & Staphylococcus grp Q1 \\
\hline 6. & $\begin{array}{l}\text { Deep zone } \\
\text { (guano soil) }\end{array}$ & кСв93 & + & Deinococcus radidurans \\
\hline 7. & $\begin{array}{l}\text { Deep zone } \\
\text { (red laterite } \\
\text { soil) }\end{array}$ & КСВ140 & + & Micrococcus agilis \\
\hline
\end{tabular}

- Micrococcus luteus3 KCB38 was used for antibody production (+ = agglutination; - = no agglutination)

ARDRA technique was applied for further confirmation of the selected bacteria. Amplified fragments of 16SrDNA were digested with EcoRI. Restriction fragment were analyzed by the using $2.5 \%$ agarose gel. In entrance zone four bacterial isolates were studied for ARDRA pattern i.e. Stomatococcus mucilaginosus KCB14, Micrococcus luteus 4 KCB11, Stomatococcus mucilaginosus KCB26, Deinococcus radiodurans KCB21. 16SrDNA of Stomatococcus mucilaginosus 
KCB14 was cut into 6 fragments i.e. 5000bp, 4000bp, 3900bp, $3000 \mathrm{bp}, 2600 \mathrm{bp}$ and 2500bp whereas in Stomatococcus mucilaginosus KCB $264000 \mathrm{bp}$ fragment is absent. Micrococcus luteus 4 KCB11 DNA was fragmented into 7 part i.e. 4500, 3800, 3500, 2400, 2300, 2200, 2000 bp, but Micrococcus luteus 3 KCB38 from transient zone observed 6 fragments 3800, 3600, 3500, 3000, 2600 and 2500bp. Deinococcus radiodurans KCB21 isolated from entrance zone and guano soil of deep zone shows same number of bands these were 4500, 3800, 3500, 2500, 2400, 2300, 2000bp. Micrococcus agilis from foval soil and alluvial soil of deep zone shows the same number of band pattern. 16SrDNA of Staphylococcus simulans KCB 129 was fragmented into 6 parts i.e. 4200, 3800, 3600, 3500, 3100, 3000 bp while Staphylococcus varians KCB134 shows 4200, 4000, 3900, 3100 and 3000bp. And Streptococcus grp Q1 KCB109 shows 4200, 3500, 2600,2500, 2400, 2200 (table 2, Figure 1\&2).

Table2: ARDRA pattern with restriction enzymes EcoRI. Sizes were expressed in bp

\begin{tabular}{|c|c|c|c|c|}
\hline $\begin{array}{l}\text { S. } \\
\text { N. }\end{array}$ & Zone & $\begin{array}{l}\text { Culture } \\
\text { code }\end{array}$ & $\begin{array}{l}\text { Bacterial } \\
\text { isolates }\end{array}$ & $\begin{array}{l}\text { EcoRI digested } 16 \mathrm{~S} \\
\text { rDNA fragment (bp) }\end{array}$ \\
\hline \multirow{4}{*}{1.} & \multirow{4}{*}{$\begin{array}{l}\text { Entrance } \\
\text { zone }\end{array}$} & KCB14 & $\begin{array}{l}\text { Stomatococcus } \\
\text { mucilaginosus }\end{array}$ & $\begin{array}{l}5000, \quad 4000, \\
3000,2600,2500\end{array}$ \\
\hline & & KCB11 & $\begin{array}{l}\text { Micrococcus } \\
\text { luteus } 4\end{array}$ & $\begin{array}{l}4500,3800,3500,2400, \\
2300,2200,2000\end{array}$ \\
\hline & & КСB26 & $\begin{array}{l}\text { Stomatococcus } \\
\text { mucilaginosus }\end{array}$ & $\begin{array}{l}5000, \quad 3800, \\
2600,2500\end{array}$ \\
\hline & & КСB21 & $\begin{array}{l}\text { Deinococcus } \\
\text { radiodurans }\end{array}$ & $\begin{array}{l}4500, \quad 3800, \\
2500,2400,2300,2000\end{array}$ \\
\hline 5. & $\begin{array}{l}\text { Twilight } \\
\text { zone }\end{array}$ & KCB38 & $\begin{array}{l}\text { Micrococcus } \\
\text { luteus } 3\end{array}$ & $\begin{array}{l}3800, \quad 3600, \\
3000,2600,2500\end{array}$ \\
\hline 6. & $\begin{array}{l}\text { Transient } \\
\text { zone }\end{array}$ & KCB50 & $\begin{array}{l}\text { Deinococcus } \\
\text { radiodurans }\end{array}$ & 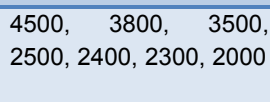 \\
\hline \multirow{3}{*}{7.} & \multirow{3}{*}{$\begin{array}{l}\text { Deep zone } \\
\text { (foval soil) }\end{array}$} & KCB129 & $\begin{array}{l}\text { Staphylococcus } \\
\text { simulans }\end{array}$ & $\begin{array}{l}4200,3800, \\
3500,3100,3000\end{array}$ \\
\hline & & KCB134 & $\begin{array}{l}\text { Staphylococcus } \\
\text { varians }\end{array}$ & $\begin{array}{l}4200, \quad 4000, \\
3100,3000\end{array}$ \\
\hline & & KCB125 & $\begin{array}{l}\text { Micrococcus } \\
\text { agilis }\end{array}$ & $\begin{array}{lll}4200, & 3500, & 2800, \\
2700, & 2500, & 2400, \\
2300, & 2000\end{array}$ \\
\hline 10. & $\begin{array}{l}\text { Deep zone } \\
\text { (guano mix } \\
\text { soil) }\end{array}$ & KCB109 & $\begin{array}{l}\text { Streptococcus } \\
\text { grpQ1 }\end{array}$ & $\begin{array}{l}4200,3500,2600,2500, \\
2400,2200\end{array}$ \\
\hline 11. & $\begin{array}{l}\text { Deep zone } \\
\text { (guano soil) }\end{array}$ & КСВ93 & $\begin{array}{l}\text { Deinococcus } \\
\text { radiodurans }\end{array}$ & $\begin{array}{lr}4500, & 3800, \\
2500,2400,2300, & 3500\end{array}$ \\
\hline 12. & $\begin{array}{l}\text { Deep zone } \\
\text { (alluvial soil) }\end{array}$ & KCB140 & $\begin{array}{l}\text { Micrococcus } \\
\text { agilis }\end{array}$ & $\begin{array}{lll}4200, & 3500, & 2800, \\
2700, & 2500, & 2400, \\
2300, & 2000\end{array}$ \\
\hline
\end{tabular}

Figure 1: DNA fingerprint pattern of bacterial isolates from cave - after EcoRI restriction analysis.
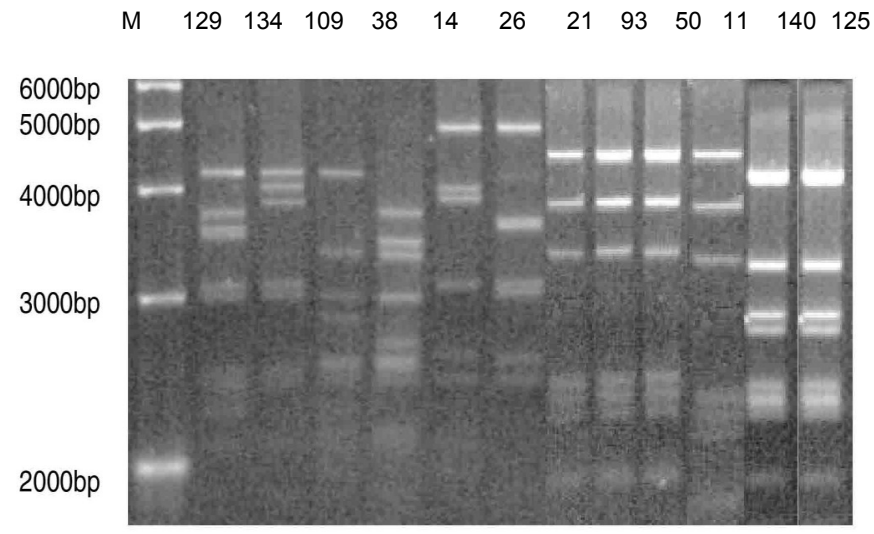

Figure 2: Dendogram showing relatedness among bacterial isolates based on restriction digestion of genomic DNA using EcoRI. Where; M: DNA Marker; 129: Staphylococcus simulans KCB129; 109: Streptococcus grp Q1 KCB109; 11: Micrococcus luteus 4 KCВ11; 134: Staphylococcus varians KСB 134, 14: Stomatococcus mucilaginosus KCB 14; 26: Stomatococcus mucilaginosus KCB26; 93: Deinococcus radiodurans KCB93; 21: Deinococcus radiodurans KCB21; 50: Deinococcus radiodurans KCB50; 38: Micrococcus luteus 3 KCB38; 140: Micrococcus agilis KCB140; 125: Micrococcus agilis KCB125.

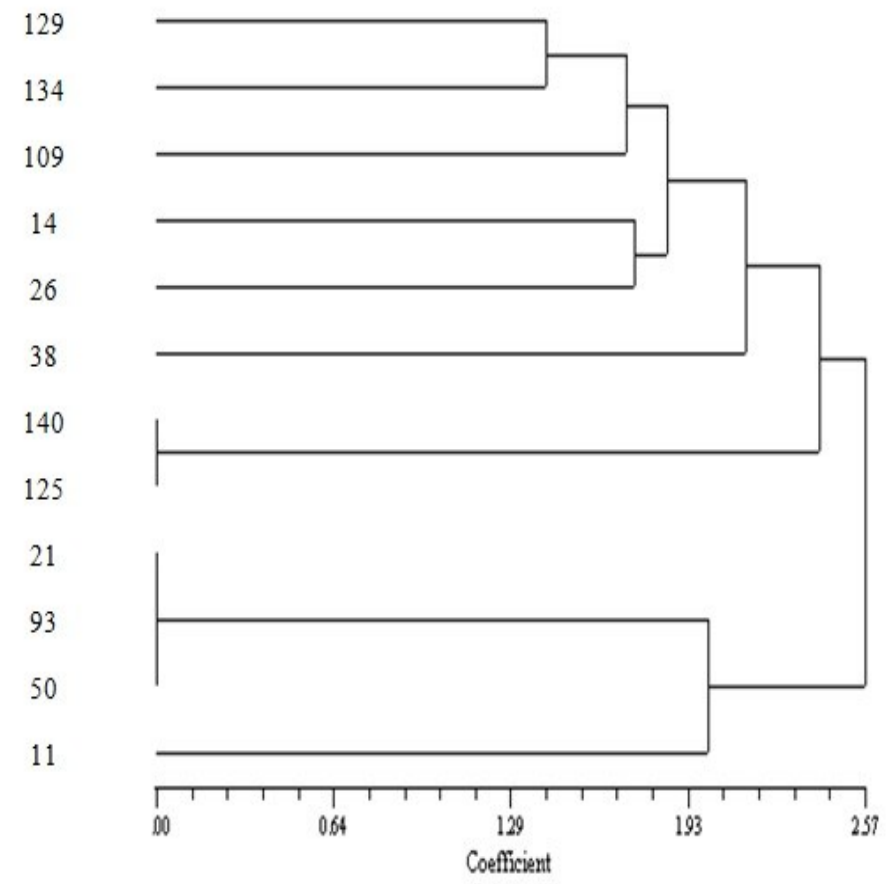

Amplified products of these bacterial 16SrDNA were also treated with Alul restriction enzyme. Stomatococcus mucilaginosus KCB14 showed 3 fragments i.e. 4900bp, 3100bp and 2900bp whereas $S$. mucilaginosus KCB26 recorded one more band that was $4800 \mathrm{bp}$ from entrance zone. Micrococcus luteus $4 \mathrm{KCB} 11$ from entrance zone showed 6000bp, 3900bp, $3100 \mathrm{bp}, 3000 \mathrm{bp}$ and 2500bp while Micrococcus luteus 3 from twilight zone KCB38 recorded 7000bp, 4300bp, 2500bp, 2400bp and 2200bp. 
Now again Deinococcus radiodurans from entrance zone, transient zone and guano soil of deep zone were show same number and size of band pattern i.e. 7000, 2900, 2600, 2500 and $2000 \mathrm{bp}$. M. agilis from foval soil and alluvial soil of deep zone recorded same pattern of bands which were 4200bp, 3300bp, $3200 \mathrm{bp}, 2500 \mathrm{bp}, 2400 \mathrm{bp}, 2300 \mathrm{bp}$ and 1800bp. Staphylococcus simulans KCB 129 exhibited 8 fragments i.e. 6000bp, 4800bp, 4200bp, 4100bp, 3200bp, 3100bp, 2500bp and 2000bp whereas Satphylococcus varians KCB134 showed only 6 fragments i.e. 4500bp, 4000bp, 3900bp, 3000bp, 2600bp and 2500bp.One isolate of guano mix soil that was Streptococcus grpQ1 showed completely different band pattern i.e. $6000 \mathrm{bp}, 5500 \mathrm{bp}, 5000 \mathrm{bp}$, 3200 bp, 3100bp, 2500 and 2000bp (table 3, Figure 3\&4).

Table 3: ARDRA pattern with restriction enzymes Alul. Sizes were expressed in bp

\begin{tabular}{|c|c|c|c|c|}
\hline $\begin{array}{l}\text { S. } \\
\text { N. }\end{array}$ & Zone & $\begin{array}{l}\text { Culture } \\
\text { code }\end{array}$ & $\begin{array}{l}\text { Bacterial } \\
\text { isolates }\end{array}$ & $\begin{array}{l}\text { Alul digested } \\
\text { 16SrDNA fragment } \\
\text { (bp) }\end{array}$ \\
\hline \multirow{4}{*}{1.} & \multirow{4}{*}{$\begin{array}{l}\text { Entrance } \\
\text { zone }\end{array}$} & KCB14 & $\begin{array}{l}\text { Stomatococcus } \\
\text { mucilaginosus }\end{array}$ & $4900,3100,2900$ \\
\hline & & KCB11 & $\begin{array}{l}\text { Micrococcus } \\
\text { luteus } 4\end{array}$ & $\begin{array}{l}6000, \quad 3900, \\
3000,2500\end{array}$ \\
\hline & & KCB26 & $\begin{array}{l}\text { Stomatococcus } \\
\text { mucilaginosus }\end{array}$ & $4900,4800,3100,2900$ \\
\hline & & KCB21 & $\begin{array}{l}\text { Deinococcus } \\
\text { radiodurans }\end{array}$ & $\begin{array}{l}7000, \quad 2900,2600, \\
2500,2000\end{array}$ \\
\hline 5. & $\begin{array}{l}\text { Twilight } \\
\text { zone }\end{array}$ & KCB38 & $\begin{array}{l}\text { Micrococcus } \\
\text { luteus } 3\end{array}$ & $\begin{array}{l}7000, \quad 4300, \quad 2500, \\
2400,2200\end{array}$ \\
\hline 6. & $\begin{array}{l}\text { Transient } \\
\text { zone }\end{array}$ & KCB50 & $\begin{array}{l}\text { Deinococcus } \\
\text { radiodurans }\end{array}$ & $\begin{array}{l}7000, \quad 2900,2600, \\
2500,2000\end{array}$ \\
\hline \multirow{3}{*}{7.} & \multirow{3}{*}{$\begin{array}{l}\text { Deep zone } \\
\text { (foval soil) }\end{array}$} & KCВ129 & $\begin{array}{l}\text { Staphylococcus } \\
\text { simulans }\end{array}$ & $\begin{array}{l}6000, \quad 4800, \quad 4200, \\
4100,3200,3100,2500, \\
2000\end{array}$ \\
\hline & & KCB134 & $\begin{array}{l}\text { Staphylococcus } \\
\text { varians }\end{array}$ & $\begin{array}{l}4500, \quad 4000, \quad 3900, \\
3000,2600,2500\end{array}$ \\
\hline & & KCB125 & $\begin{array}{l}\text { Micrococcus } \\
\text { agilis }\end{array}$ & $\begin{array}{lrr}4200, & 3300, & 3200 \\
2500,2400,2300, & 1800\end{array}$ \\
\hline 10. & $\begin{array}{l}\text { Deep zone } \\
\text { (guano mix } \\
\text { soil) }\end{array}$ & KCB109 & $\begin{array}{l}\text { Streptococcus } \\
\text { grpQ1 }\end{array}$ & $\begin{array}{l}6000, \quad 5500, \quad 5000, \\
3200,3100,2500,2000\end{array}$ \\
\hline 11. & $\begin{array}{l}\text { Deep zone } \\
\text { (guano soil) }\end{array}$ & КСВ93 & $\begin{array}{l}\text { Deinococcus } \\
\text { radiodurans }\end{array}$ & $\begin{array}{l}7000, \quad 2900,2600, \\
2500,2000\end{array}$ \\
\hline 12. & $\begin{array}{l}\text { Deep zone } \\
\text { (alluvial soil) }\end{array}$ & KCB140 & $\begin{array}{l}\text { Micrococcus } \\
\text { agilis }\end{array}$ & $\begin{array}{l}4200,3300, \quad 3200 \\
2500,2400,2300,1800\end{array}$ \\
\hline
\end{tabular}

Figure 3: DNA fingerprint pattern of bacterial isolates from cave - after Alul restriction analysis.
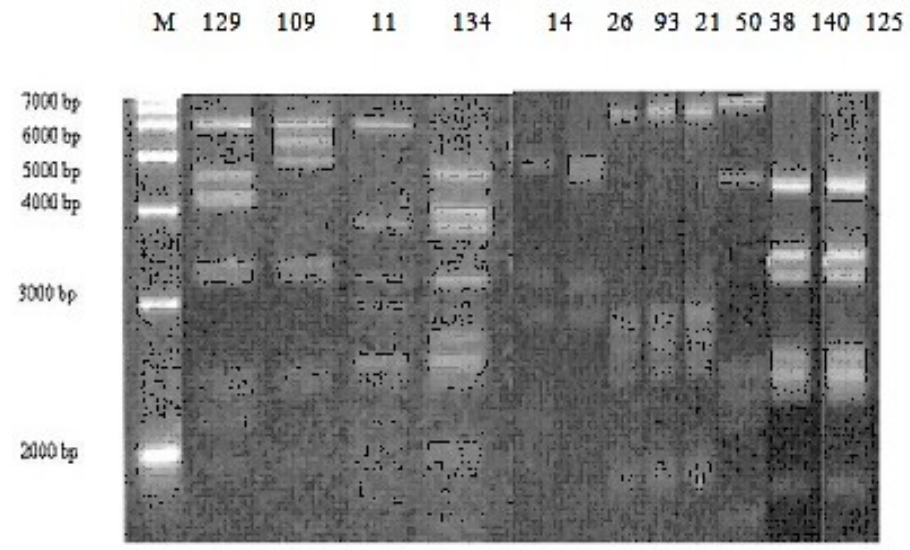

Figure 4: Dendogram showing relatedness among bacterial isolates based on restriction digestion of genomic DNA using Alul.Where; M: DNA Marker; 129: Staphylococcus simulans KCB129; 109: Streptococcus grp Q1 KCB109; 11: Micrococcus luteus 4 KCB11; 134: Staphylococcus varians $K C B$ 134, 14: Stomatococcus mucilaginosus KCB 14; 26: Stomatococcus mucilaginosus KCB26; 93: Deinococcus radiodurans KCB93; 21: Deinococcus radiodurans KCB21; 50: Deinococcus radiodurans KCB50; 38: Micrococcus luteus 3 KCB38; 140: Micrococcus agilis KCB140; 125: Micrococcus agilis KCB125.

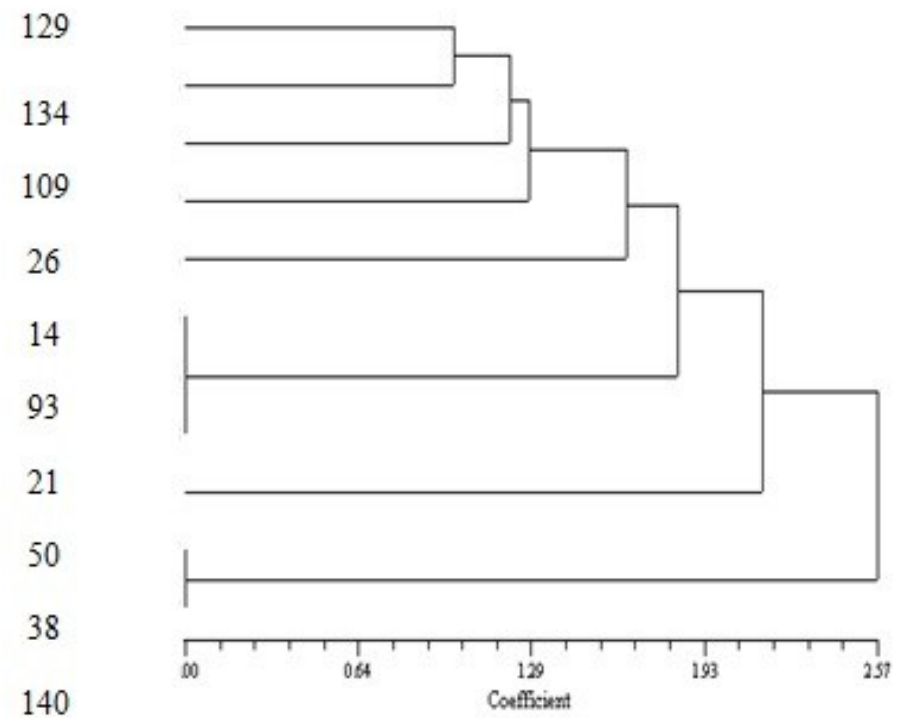

\section{Discussion}

Our earlier reports have already revealed that Stomatococcus mucilaginosus, Micrococcus luteus, Staphylococcus simulans, Staphylococcus epidermis were isolated from soil sediment samples of Kotumsar cave are pathogenic. Semenov, 1990 [13] was also isolated some human pathogens i.e. Staphylococcus, Enterobacteria, Penicillium, Aspergillus, Gymenoascaceae fungi from the Kap-Kutan and Tash-Yurak caves (Kugitangtow ridge, Turkmenistan, Russia). Jurado et al., 2010 [3] also reported some pathogenic bacteria in cave ecosystem. The conventional pathogenic microorganisms found in the caves could be dangerous for human. PIB-win identified bacteria were further confirmed by conventional method that was serotyping as well as advanced method such as ARDRA. Both techniques support the PIB-win result.

In serological test agglutination would be a useful quick screening test for sorting out Micrococci sp. from other bacterial isolates from Kotumsar cave. Antibody was produced against the surface antigen of the Micrococcus luteus3. Therefore only particulate antigen showed agglutination with antisera. Serological techniques measure similarities only at the surface of proteins and it is at the protein surface that the greatest number of antigenic sites per protein molecule. Nevertheless, serological techniques of this kind provide a rapid and convenient method for assessing structural similarities between homologous proteins, are useful in the classification of bacteria, and can also cast some light on possible phylogenetic 
relationships. Lind et al. 1996 [5] reported serotyping as a useful tool in most epidemiological situations but sometimes lack sufficient discriminatory power. DNA fingerprinting can add valuable epidemiological information to that supplied by serotyping. Makrai et al., 2005 [6] reported the plasmid types and serotypes of 164 Rhodococcus equi strains obtained from submaxillary lymph nodes of swine from different piggeries in 28 villages and towns located throughout the Hungary. Turkyilmaz, 2005 [15] studied the isolation of Ornithobacterium rhinotracheale from poultry in Turkey and serotyping of the isolates and field sera by agar gel precipitation (AGP) tests.

Amplified rDNA restriction and confirmation analysis is a useful method for confronting isolates undergoing condition changes or sample from different environment [11]. In the present study, ARDRA pattern of bacterial isolates was analyzed using universal primer and endonuclease enzymes EcoRI and Alul. The Dendogram generated on the basis ARDRA pattern using EcoRI showed that three isolates of Deinococcus radiodurans, each from entrance zone, twilight zone and deep zone (guano soil) recorded resembled each other, likewise Micrococcus agilis KCB125 from foval soil and Micrococcus agilis KCB140 from redlaterite soil of deep zone exhibited $100 \%$ similarity. However, the Micrococcus luteus 4 and Micrococcus luteus 3 were present in different clades. Ingrassia et al. [2] reported ARDRA patterns with restriction enzymes, For Stomatococcus mucilaginosus Haelll -313 287 146; Rsal- 356252147 123; Taql- 445 360; for Deinococcus radiodurans Haelll - 458 373, Rsal-502 240, Taql- 439 360; Micrococcus varians Haell- 378316 146; Rsal- 252205154 147; Taqi- 444 363. Imperi et al. 2007 [1] isolated and characterized the bacterial community from cave of Itly by ARDRA technique.

Stomatococcus mucilaginosus KCB26 and KCB14 shown same biochemical and physiological properties but there were variation in band pattern with restriction enzymes Eco RI and Alul but similar band pattern shown in case of Deinococcus radiodurans isolated from three different zone i.e. entrance, Transient as well as deep zone of guano soil. Conclusively this piece of work confirms the existence of some pathogenic bacteria like Stomatococcus mucilaginosus, Staphylococcus varians, Micrococcus luteus, Staphylococcus simulans in Kotumsar cave by serological and $16 \mathrm{~S}$ rRNA gene based technique.

\section{Conclusion}

ARDRA is a one most important tool of species analysis. In summary, this piece of work is useful for estimating the phylogenetic diversity and composition of bacterial community in oligotrophic environmental niche which is supported by serotyping. The ARDRA based approach with cluster analysis of DNA fingerprinting patterns applied in our study has been demonstrated to be effective in possessing new data concerning the biospeleological study. Our molecular and serological confirmation of bacteia supports the previous findings [8\&9] this study has shown the presence of some of the pathogenic bacteria in cave environment. Hence proper safety precautions like appropriate hand washing, changing the clothes and shoes are recommended for the cave workers and visitors for prevention of pathogenic threat to them.

\section{Acknowledgement}

First author is thankful to the Dr. A.Gupta Professor and Head, School of Studies in Life Sciences, Pt. Ravishankar Shukla University, Raipur, Chhattisgarh, India for providing lab facility and conduct the serological work. Author expresses her deep sense of gratitude and indebtedness to Prof. Anjana Sharma, Professor, Department of Bioscience, Rani Durgawati University, Jabalpur, (M.P.), India for her most valuable guidance, keen interest, encouragement and suggestion throughout this investigation.

\section{Reference}

1. Imperi F, Caneva G, Cancellieri L, Ricci MA, Sodo A, Visca $P$. The bacterial aetiology of rosy discoloration of ancient wall paintings. Environmental Microbiology. 2007; 9(11): 2894-2902.

2. Ingrassia I, Roques C, Prevots F, Preliminary experiments for ARDRA validation on flora associated with intestinal mucosa: EDP Sciences. 2001; 263-280.

3. Jurado V., Laiz L, Rodriguez-Nava V, Boiron P, Hermosin B, Sanchez-Moral S, Saiz-Jimnez C. Pathogenic and opportunistic microorganisms in caves. International Journal of Speleology. 2010; 39 (1): 15-24.

4. Lagace L, Pitre M, Jacques M, Roy D. Identification of the Bacterial Community of Maple Sap by Using Amplified Ribosomal DNA (rDNA) Restriction Analysis and rDNA sequencing. Applied and Environmental Microbiology. 2004; 70 (4): 2052-2060.

5. Lind L, Sjogren E, Melby K, Kaijser B. DNA Fingerprinting and serotyping of Campylobacter jejuni isolates from epidemic outbreaks. Journal of clinical Microbiology 1996; 34(4): 892-896.

6. Makrai L, Takayama S, Denes B, Istvan H, Sasaki $Y$, Kakuda T, Tsubaki S, Major A, Fodor L, Verga J, Takai S. Characterizion of Virulence Plasmid and Serotyping of Rhodococcus equi isolates from submaxillary lymph nodes of pigs in Hungry. Journal of clinical Microbiology. 2005; 43 (3): 1246-1250.

7. Prescott JF.. Capsular Serotypes of Corynebacterium equi . Can. J. comp. Med. 1981; 45: 130-134.

8. Rajput Y, Biswas J. Subterranean depth dependent protein constitutions of the Micrococcus sp., isolated from the Kotumsar cave, India. Asian Journal of Biochemistry. 2012; 7(2): 90-97. 
9. Rajput Y, Rai V, Biswas J. Screening of Bacterial isolates from various Microhabitat sediments of Kotusar cave: A cogitation on their Respective Benefits and expected threats for complete biosphere and tourists. Research Journal of Environmental Toxicology. 2012; 6 (1):13-24.

10. Rajput Y, Neral A, Biswas J. Subterranean Depth Dependent the Modulation of Endo and Exoenzyme Secretion in Streptomyces prasinosporus: A common soil Actinomycetes of India. Journal of Pure and Applied Microbiology. 2014; 8 (6): 4601-4608.

11. Sarra PG, Zacconi C,Scolari G, Characterization of specific microflora involved in "Culatello" ripening. Annals of Microbiology. 2004; 54 (1): 49-58.

12. Sambrook J, Fritsh EF, Maniatis T. Molecular cloning: A laboratory Manual, Cold Spring Harbor Lab Press, Plainview, New York 1989.

13. Semenov SM. Laboratory media for actinomycetes and fungi: Moscow. 1990; 172.

14. Thorn G, Tsuneda A. Molecular genetic characterization of bacterial isolates causing brown blotch on cultivated mushrooms in Japan. Mycoscience. 1996; 37: 409-16.

15. Turkyilmaz S. Isolation and serotyping of Ornithobacterium rhinotracheale from poultry. Turk J Vet Anim Sci. 2005; 29: 1299-1304. 